\title{
Solvent Effect and Two-Photon Optical Properties of Triphenylamine-Based Donor-Acceptor Fluorophores
}

Yilin Zhang ${ }^{1}$, Meijuan Jiang ${ }^{2}$, Guang-Chao Han ${ }^{3}, \mathrm{Ke} \mathrm{Zhao}^{4}$, Ben Zhong Tang ${ }^{2}$, and Kam Sing Wong $^{1, *}$

${ }^{1}$ Department of Physics, the Hong Kong University of Science and Technology, Clear Water Bay, Hong Kong, China

${ }^{2}$ Department of Chemistry, the Hong Kong University of Science and Technology, Clear Water Bay, Hong Kong, China

${ }^{3}$ Beijing National Laboratory for Molecular Sciences, CAS Key Laboratory of Organic Solids, Institute of Chemistry, Chinese Academy of Sciences, Beijing, China, 100190

${ }^{4}$ College of Physics and Electronics, Shandong Normal University, Jinan, China, 250014 *phkswong@ust.hk (K.S.W.)

\section{Solvent parameters}

Table S 1 Some physical properties of the solvents discussed in the article:

\begin{tabular}{llll}
\hline Solvent & $\varepsilon$ & $n$ & $\Delta f$ \\
Hexane & 1.89 & 1.38 & -0.0019 \\
Toluene & 2.38 & 1.5 & 0.0123 \\
Et2O & 4.33 & 1.35 & 0.1676 \\
Dioxane & 2.25 & 1.42 & 0.0253 \\
THF & 7.58 & 1.41 & 0.2086 \\
EA & 6.02 & 1.37 & 0.2005 \\
Acetone & 20.7 & 1.36 & 0.2838 \\
MeCN & 37.5 & 1.34 & 0.307 \\
DMSO & 46.7 & 1.48 & 0.2629 \\
\hline
\end{tabular}


in which $\Delta f=\frac{\varepsilon-1}{2 \varepsilon+1}-\frac{n^{2}-1}{2 n^{2}+1}$ is the quantity to describe the solvent polarity in the general Onsager's reaction field theory. The solutions are sorted in ascending order of emission peak wavelength of the fluorophores inside studied.

\section{Experiments}

\subsection{Instrumentation}

\section{- UV visible absorption and emission spectra}

The UV absorption spectra were measured using a Biochrom Libra spectrometer. The onephoton fluorescence spectra were scanned with a Perkin Elmer LS55 spectrofluorometer. All the solutions are with a fluorophore concentration of $10 \mu \mathrm{M}$ in $1 \mathrm{~cm}$-thick quartz cell for the absorption and $40 \mu \mathrm{M}$ emission characterizations mentioned above.

\section{- Method to obtain LE/TICT state}

The method for obtaining the LE/TICT spectra is as follows: in case the spectra intensity at either shorter wavelength side $(\sim 500 \mathrm{~nm})$ or longer wavelength side $(\sim 800 \mathrm{~nm})$ are normalized to the same intensity, then comparison should be made regarding the difference in intensity of the PL spectra on the other side under different excitation wavelengths. For example, if the intensity at the short wavelength side (mainly assigned to LE emission) for TPA-BMO in acetone is normalized to the same intensity for spectra under 410 and $420 \mathrm{~nm}$ excitation, the spectra difference at the longer wavelength side is attribute to the extra TICT emission.. 

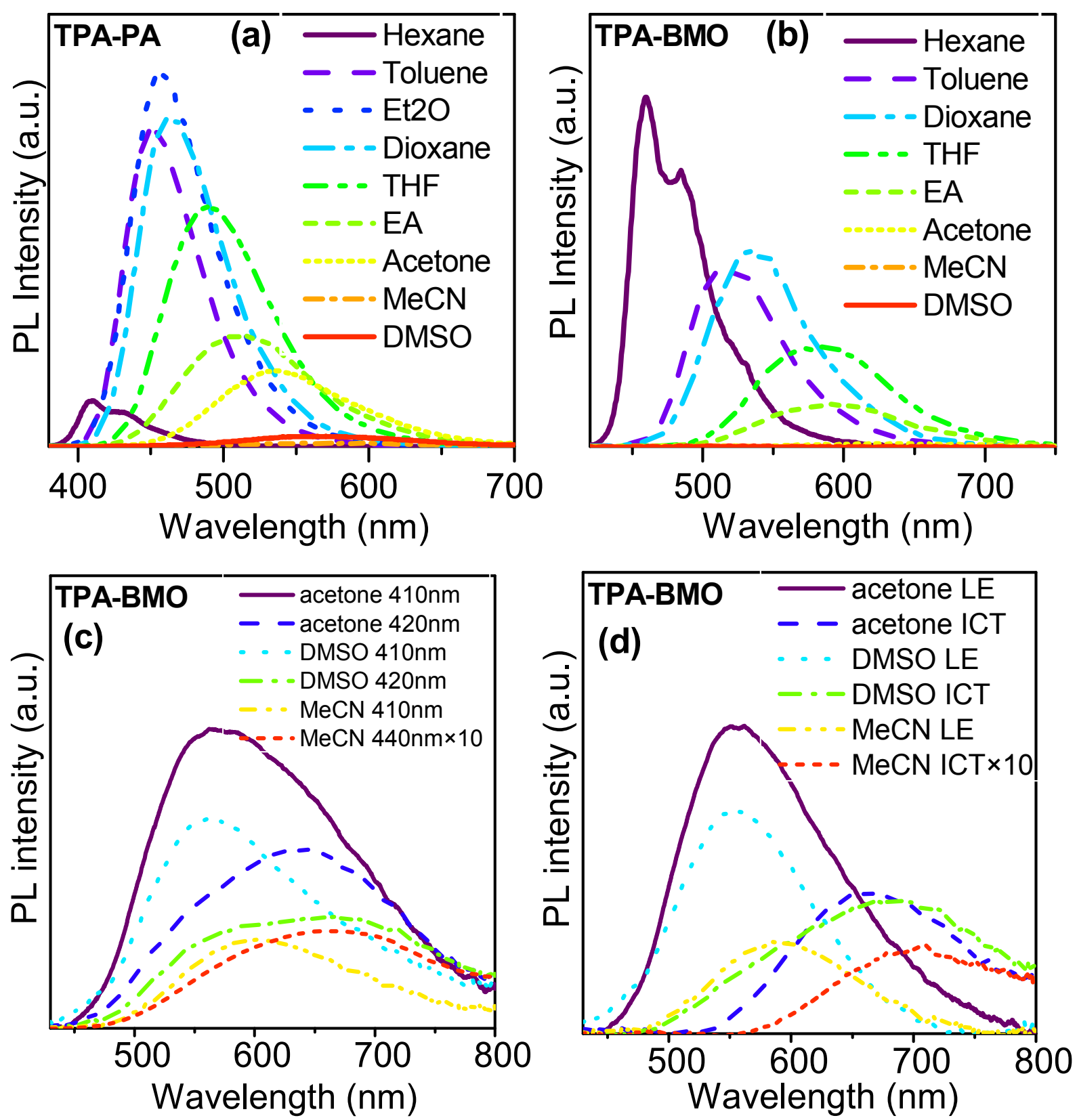

Fig. S 1 Steady-state one-photon fluorescence of (a) TPA-PA under $380 \mathrm{~nm}$ excitation and (b) TPA-BMO under $420 \mathrm{~nm}$ excitation in various solvents. Dye concentration: $10 \mu \mathrm{M}$. (c) Onephoton fluorescence emission spectra for TPA-BMO in strongly polar solvent under 410, 420 and $440 \mathrm{~nm}$ SHG excitation. Dye concentration: $40 \mu \mathrm{M}$. (d) Spectra of the two emission states (probable LE and TICT states) for TPA-BMO in strongly polar solvents. 


\section{- Fluorescence quantum efficiency}

The fluorescence quantum efficiencies were measured using a calibrated integrating sphere (Labsphere) together with a Ocean Optics USB2000 spectrometer. ${ }^{1,2}$ The excitation source for fluorescence was a second harmonic generation (SHG) from femtosecond Ti: Sapphire laser pulses in a LBO crystal. Unless otherwise specified, this SHG was tuned to $380 \mathrm{~nm}$ with a power of $0.5 \mathrm{~mW}$ for TPA-PA solutions and $420 \mathrm{~nm} 1 \mathrm{~mW}$ SHG for TPA-BMO solutions. All the solutions were measured in a quartz cell with a chromophore concentration of $40 \mu \mathrm{M}$.

\section{- Fluorescence decay and time-resolved emission spectra}

The fluorescence decays were captured with an excitation of $400 \mathrm{~nm}$ second harmonic generation (SHG) from a Ti:Sapphire oscillator (Coherent Mira 900), and collected after a longpass filter with a SpectroProf275 and a TCSPC system (The bh GmbH). The time-resolved fluorescence spectra were captured using a Hamamatsu streak camera with an excitation of 400 nm SHG from a Ti:Sapphire oscillator.
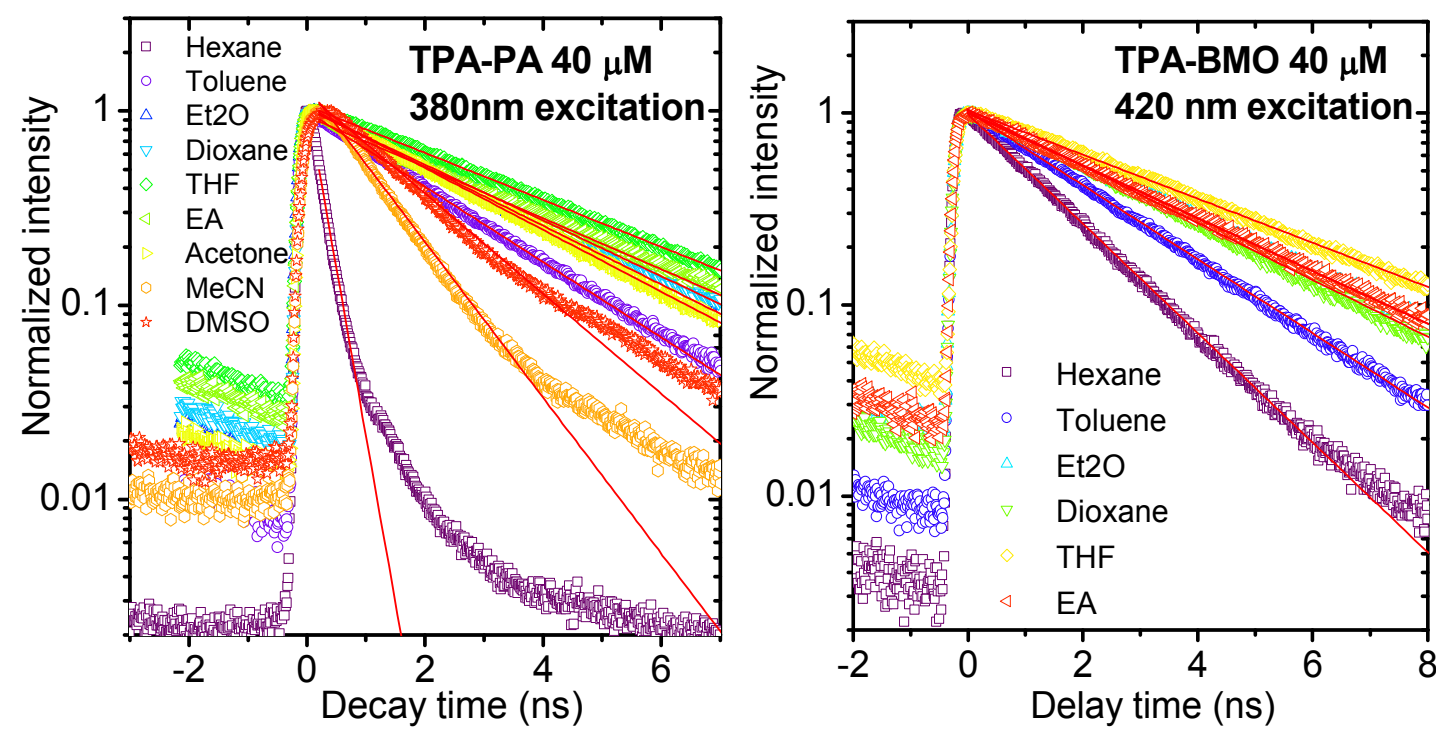

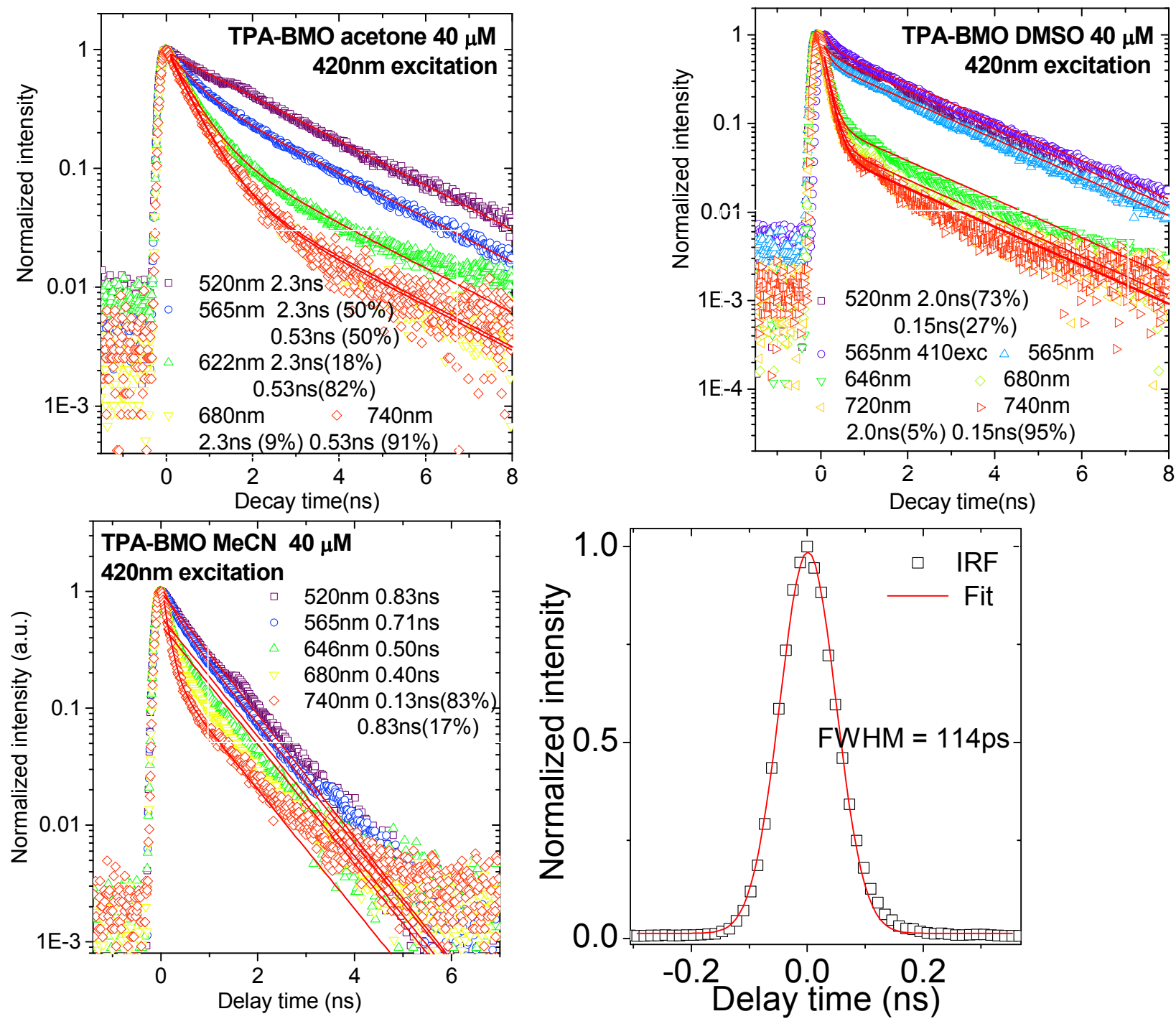

Fig. S 2 PL decay for these molecules in various solvent environments. Unless wavelength is otherwise specified, the decays are captured at their fluorescence peak wavelength. The last graph indicates the instrumental response function (IRF is related to the time resolution limit of the TCSPC system used.

\section{- Two-photon excited fluorescence (TPEF)}

The TPEF was excited with femto-second pulses with a repetition rate of $76 \mathrm{MHz}$ from a Ti:Sapphire oscillator, and then detected with a spectrometer (SpectraPro275) coupled to a 
photomultiplier tube with a lock-in system. The TPEF emission spectra of the TPA-based fluorophores studied in various solvents were captured under the same excitation conditions with 2PA standard Fluorescein in $\mathrm{PH}=11$ solution, whose $2 \mathrm{PA}$ cross section was acquired from the site : www.drbio.cornell.edu/cross_sections.html, and then corrected with an instrumental spectral response function determined with a calibrated tungsten halogen lamp, to reduce the effect of instrumental spectral response on the fluorescence collection efficiency. After the TPEF emission spectra of the sample and 2PA standard were captured, TPEF and 2PA cross-sections are estimated using the following equations $\sigma_{T P E F},=\left(F_{S} / F_{r e f}\right)\left(c_{r e f} / c_{S}\right) \sigma_{T P E F, \text { ref }}$ and $\sigma_{2 P A}=$ $\sigma_{T P E F} / \phi{ }^{3}$ where $\sigma_{T P E F}$ and $\sigma_{2 P A}$ are TPEF and 2PA cross-sections respectively, subscript $s$ refers to the quantities of sample solutions while ref stands for those of the reference, $F$ refers to the integrated intensity of fluorescence spectra after being corrected with the instrumental spectral response function, and $c$ stands for the molar concentration of fluorophores in the sample solutions. $\phi$ is one-photon excited fluorescence quantum efficiency, which is used here under the assumption that the fluorescence quantum efficiencies involved in one- and two-photon excited fluorescence are identical. 


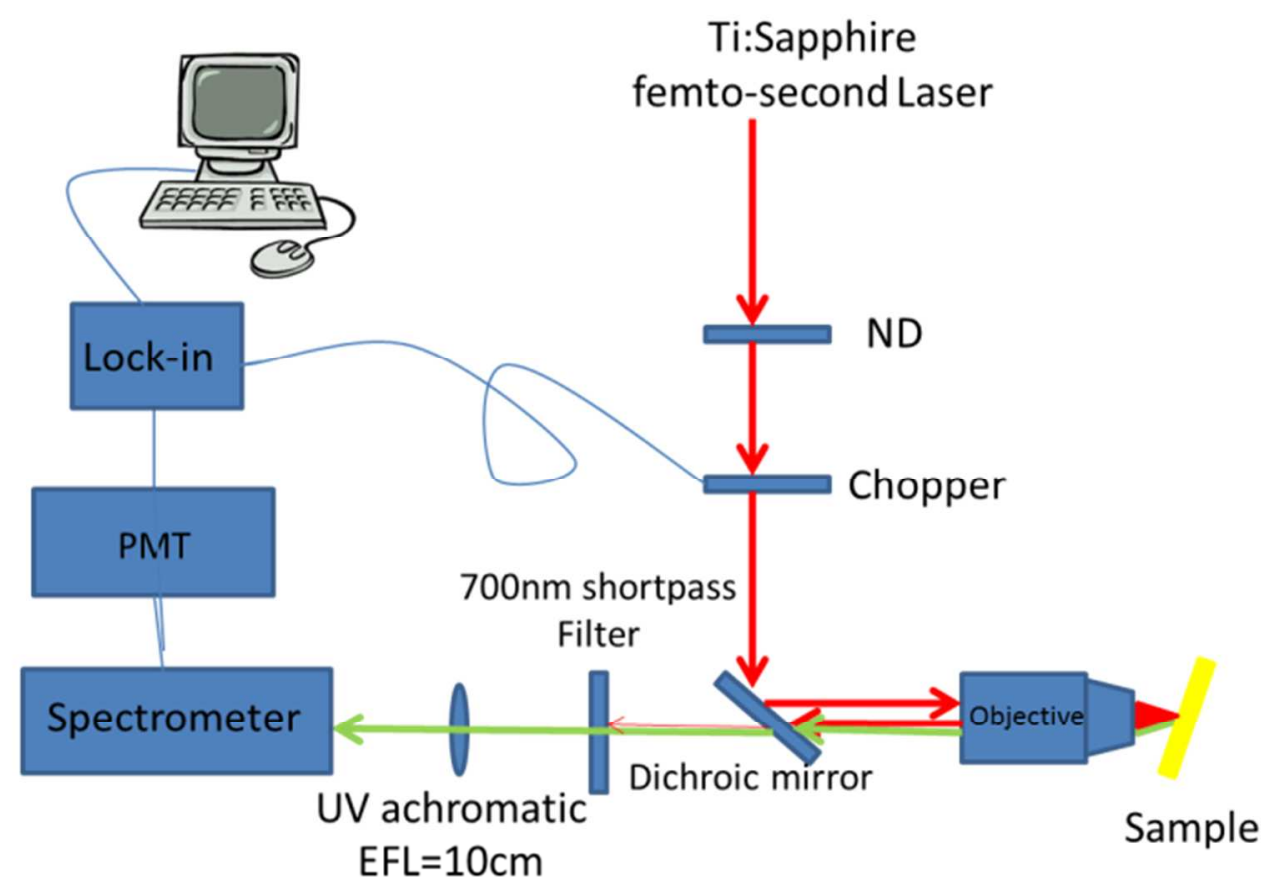

Fig. S 3 Diagram of experimental setup of the TPEF. In this diagram, the dichroic mirror is a Semrock $670 \mathrm{~nm}$ edge BrightLine multiphoton short-pass dichroic beam splitter, the objective is $10 \mathrm{X} \mathrm{NA}=0.25$, the samples measured are in a $2 \mathrm{~mm}$-thick quartz cell.

To verify the nature of the absorption process, their two-photon-excited fluorescence were examined with a Ti: Sapphire excitation around double of the UV absorption peak wavelengths. The relation between TPEF intensity and incident laser power is demonstrate in Fig. S4, a quadratic dependence is clearly seen, indicating the absorption process involved is $2 \mathrm{PA}$. 


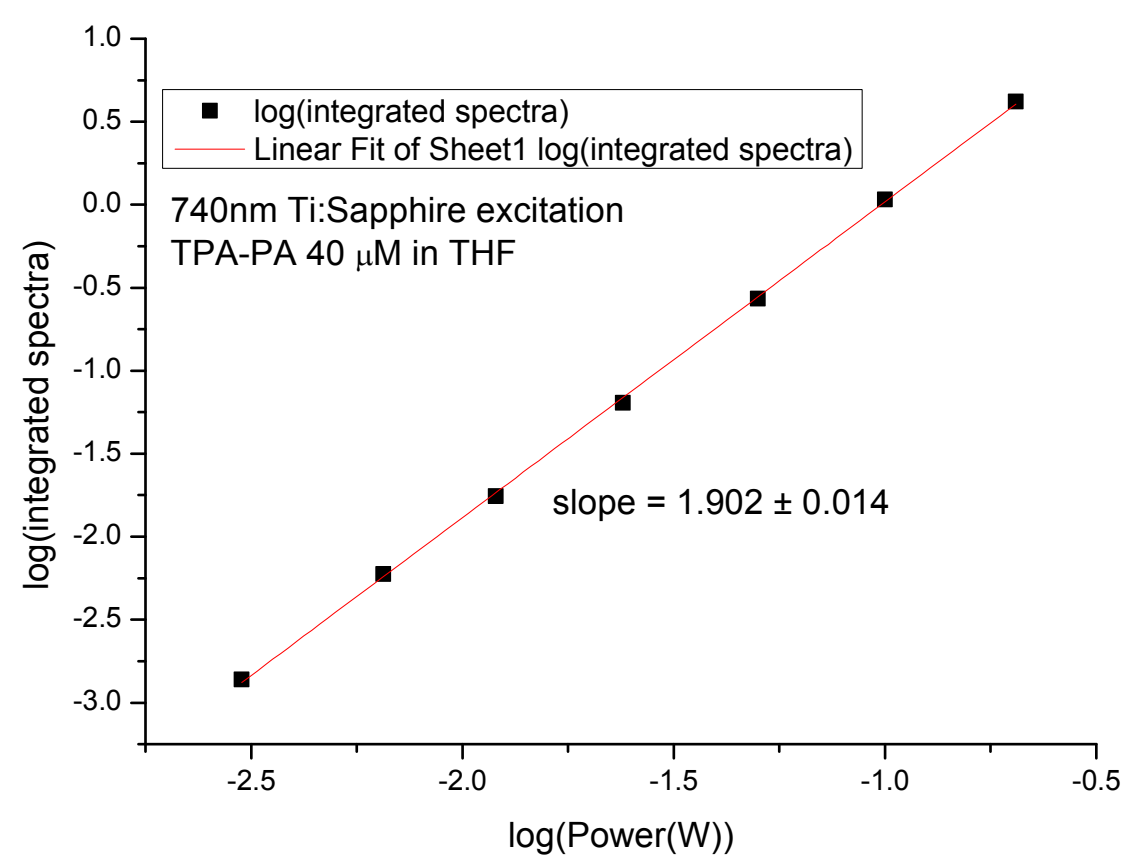

Fig. S 4 Power dependence of TPEF of TPA-PA THF solution

\subsection{Quantum chemical calculation:}

The geometries of TPA-PA and TPA-BMO molecules in solvents are optimized at hybrid B3LYP level with the $6-31+\mathrm{G}(\mathrm{d}, \mathrm{p})$ basis set in the Gaussian 09 program. ${ }^{4}$ The solvent effects are taken into account within the polarizable continuum model (PCM). The Onsager radii are determined by the molecular volume calculated by Gaussian. The time-dependent density functional theory (TD-DFT) is employed to obtain the transition dipole moments from the ground to the first excited state. The 2PA cross-sections, as well as the permanent dipole moments of the excited states are calculated through a residue of quadratic response function using the CAMB3LYP functional with 6-31G(d) basis set in the Dalton program. ${ }^{5}$

\subsection{Material information}

\section{- Material Synthesis}


TPA-PA was synthesized using Suzuki coupling reaction. Briefly, 4-bromo-N,Ndiphenylaniline(1.29 g, $4 \mathrm{mmol})$, (4-formylphenyl) boronic acid (0.66 g, $4.4 \mathrm{mmol}), \mathrm{Pd}_{\left(\mathrm{PPh}_{3}\right)_{4}}$ $(140 \mathrm{mg}), \mathrm{K}_{2} \mathrm{CO}_{3}(1.38 \mathrm{~g}, 10 \mathrm{mmol})$ in $50 \mathrm{~mL}$ of THF/water $(4: 1 \mathrm{v} / \mathrm{v})$ were refluxed overnight under nitrogen. After cooling to room temperature, water was added and the mixture was extracted with dichloromethane. After removing the solvent, the crude product was further purified with a silica gel column. A yellow solid was obtained with $75 \%$ yield.

Synthesis of TPA-BMO:349 mg (1 mmol) TPA-PA, $234 \mathrm{mg}(2 \mathrm{mmol}) \mathrm{N}$-acetylglycine, and $164 \mathrm{mg}(2 \mathrm{mmol}) \mathrm{NaOAc}$ in $4 \mathrm{~mL}$ of acetic anhydride were heated in a $100^{\circ} \mathrm{C}$ oil bath for $4 \mathrm{~h}$. After the solvent was removed, the crude product was purified by silica gel column chromatography with ethyl acetate/ hexane as eluent. A red solid was obtained with 54\% yield.

\section{- Material preparation}

To prepare for the one- and two-photon optical measurements, the TPA-PA and TPA-BMO were dissolved in distilled THF with a concentration of $4 \mathrm{mM}$ as stock solutions. One way to make the dilute solutions for the optical measurements is drying $10 \mu L$ of stock solution with condensed air flow, and then adding $1 \mathrm{~mL}$ of corresponding solvents to dissolve the concentrated fluorophores again. After the resolvation, fluorophore solutions with a concentration of $40 \mu M$ were made, which are the solutions discussed in the following content unless otherwise stated. Absorption and emission spectra were measured immediately after the solutions were made.

\section{- Material characterization}

Characterization data of TPA-PA: ${ }^{1} \mathrm{H}-\mathrm{NMR}\left(400 \mathrm{MHz} ; \mathrm{CDCl}_{3}\right) \delta_{\mathrm{H}} 10.02(\mathrm{~s}, 1 \mathrm{H}), 7.92(\mathrm{~d}, 2 \mathrm{H}$, $J=8.4 \mathrm{~Hz}), 7.72(\mathrm{~d}, 2 \mathrm{H}, J=8.0 \mathrm{~Hz}), 7.51(\mathrm{~d}, 2 \mathrm{H}, J=8.0 \mathrm{~Hz}), 7.31-7.27(\mathrm{~m}, 4 \mathrm{H}), 7.15-7.13(\mathrm{~m}$, 6H), 7.08-7.05 (m, 2H) ppm. ${ }^{13} \mathrm{C}-\mathrm{NMR}\left(400 \mathrm{MHz} ; \mathrm{CDCl}_{3}\right) \delta_{\mathrm{C}} 192.1,148.6,147.5,146.8,134.9$, 
133.0, 130.5, 129.6, 128.2, 127.1, 125.1, 123.7, 123.3. MALDI-MS calculated for $\mathrm{C}_{25} \mathrm{H}_{19} \mathrm{NO}$ $[\mathrm{M}+\mathrm{H}]^{+}$350.1539, found 350.1541.

Characterization data of TPA-BMO: ${ }^{1} \mathrm{H}-\mathrm{NMR}\left(400 \mathrm{MHz} ; \mathrm{CDCl}_{3}\right) \delta_{\mathrm{H}} 8.12(\mathrm{~d}, 2 \mathrm{H}, J=8.4 \mathrm{~Hz})$, $7.65(\mathrm{~d}, 2 \mathrm{H}, J=8.4 \mathrm{~Hz}), 7.51(\mathrm{~d}, 2 \mathrm{H}, J=8.4 \mathrm{~Hz}), 7.30-7.25(\mathrm{~m}, 4 \mathrm{H}), 7.17-7.12(\mathrm{~m}, 7 \mathrm{H})$, 7.08-7.04 (m, 2H), $2.41(\mathrm{~s}, 3 \mathrm{H}) \mathrm{ppm} .{ }^{1} \mathrm{H}-\mathrm{NMR}\left(400 \mathrm{MHz} ; \mathrm{CD}_{2} \mathrm{Cl}_{2}\right) \delta_{\mathrm{H}} 8.15(\mathrm{~d}, 2 \mathrm{H}, J=8.0 \mathrm{~Hz})$, $7.68(\mathrm{~d}, 2 \mathrm{H}, J=8.4 \mathrm{~Hz}), 7.55$ (d, 2H, $J=8.4 \mathrm{~Hz}), 7.31-7.28(\mathrm{~m}, 4 \mathrm{H}), 7.14-7.05$ (m, 9H), $2.40(\mathrm{~s}$,

$3 \mathrm{H})$ ppm. ${ }^{13} \mathrm{C}-\mathrm{NMR}\left(400 \mathrm{MHz} ; \mathrm{CD}_{2} \mathrm{Cl}_{2}\right) \delta_{\mathrm{C}} 168.4,166.6,148.7,148.0,143.7,133.7,133.3$, $132.9,132.3,131.1,129.9,128.3,127.2,125.3,123.9,123.7,16.0 \mathrm{ppm}$. MALDI-MS calculated for $\mathrm{C}_{29} \mathrm{H}_{22} \mathrm{~N}_{2} \mathrm{O}_{2}[\mathrm{M}+\mathrm{H}]^{+}$431.1754, found 431.1775.

\section{References}

(1) de Mello, J. C.; Wittmann, H. F.; Friend, R. H. An Improved Experimental Determination of External Photoluminescence Quantum Efficiency. Adv. Mater. 1997, 9, 230 232.

(2) Tam, M. C.; Su, H.; Wong, K. S.; Zhu, X.; Kwok, H. S. Surface-PlasmonEnhanced Photoluminescence from Metal-Capped Alq3 Thin Films. Appl. Phys. Lett. 2009, 95, 051503 .

(3) Makarov, N. S.; Drobizhev, M.; Rebane, A. Two-Photon Absorption Standards in the 550-1600 Nm Excitation Wavelength Range. Opt. Express. 2008, 16, 4029-4047.

(4) Frisch, M. J.; Trucks, G. W.; Schlegel, H. B.; Scuseria, G. E.; Robb, M. A.; Cheeseman, J. R.; Scalmani, G.; Barone, V.; Mennucci, B.; Petersson, G. A.et al.; A. 01 ed.; Gaussian, Inc.: Wallingford, CT, USA, 2009.

(5) Aidas, K.; Angeli, C.; Bak, K. L.; Bakken, V.; Bast, R.; Boman, L.; Christiansen, O.; Cimiraglia, R.; Coriani, S.; Dahle, P.et al. The Dalton Quantum Chemistry Program System. Wiley Interdisciplinary Reviews: Computational Molecular Science. 2013, 4, 269-284. 